ceed in removing the foreign body. After drying the any other cavity within the body, would in a greater tube a fresh supply of absorbent cotton is placed or lesser degree retard the progress of the case toward over its mouth and the corners of the rubber dam recovery, and in many instances endanger, if not sacneatly folded over it and pinned. This same precau- rifice the life of the patient. I do not believe that it tion should be observed at each cleansing of the tube. is necessary, or even advisable, to use drainage in The tube should be rotated and raised a quarter of every case of abdominal section. If aseptic condian inch two or three times for the first. twenty-four tions are scrupulously maintained throughout the hours. This relieves pressure on the intestines, if operation, and all bleeding vessels secured, the abdomany exist and at same time prevents the omentum inal cavity thoroughly irrigated with pure distilled becoming entangled in the holes of the tube, an ac- water (that is warmed and has previously been thorcident more of an anticipated dread than of actual oughly boiled), whenever any blood or foreign suboccurrence. After removing the glass tube if there stance has escaped into it-under these circumstances is any fluid likely to collect within the next twenty- drainage is not necessary; and if not necessary, it is four hours, a small rubber tube may be left in the certainly not advisable. It should not be lost sight of site of the glass tube by introducing it through the for one moment that the drainage tube is at best, a forglass tube before it is removed. This in turn can eign body, and capable of causing a good deal of irritausually be removed on the following day.

By using drainage as I have described, the bandages and dressings are kept dry and clean, do not have to be disturbed until the time arrives to remove the tube or stitches. When the tube fails to show much over a drachm of fluid, collected in an hour or two, the time has arrived to remove it, unless this small amount of fluid is purulent or offensive. Ordinarily the tube can be removed with safety on the second or third day. The opening soon closes and there is no more danger of a ventral hernia fol. lowing than if drainage had not been used.

Drs. Price and Wylie years ago called attention to the fact that many deaths from so-called shock were in reality deaths from concealed fatal hæmorrhages and advocated the introduction of the tube in these cases in which there was free oozing during or following an operation. The presence of the tube, by keeping the peritoneal cavity free from lymph is a direct and most efficient hæmostatic. This fluid normally present in small amount is greatly augmented by the manipulation of the peritoneum during the performance of an abdominal section, and like the moist fomentations over the scarrified surface of any other part of the body, promotes bleeding and prevents the formation of firm clots in the mouths of the small blood vessels torn across while breaking down adhesions.

Gentlemen, if you will use "Peritoneal Irrigation and Drainage" as I have described it, you will not,I am sure, condemn the method. They will help reduce your mortality, I am sure.

1016 East 9th Street.

\section{DRAINAGE IN ABDOMINAL SURGERY.}

Read in the Section of Obstetries and Diseases of Women, at the Fort third Annual Meeting of the American Medical Association, held at
Detroit, Mich., June, 1892.

BY DONNEL HUGHES, M.D.,

OF PHILADELPHIA, PA.

Drainage of the peritoneal cavity is one of the most important subjects connected with abdominal surgery, and yet at the same time, it is one of the most disputed points. The difference of opinion that exists to so marked a degree is due, no doubt, to the fear that the tube may act as a source of septic infection, or by its use the abdominal walls are weakened to such an extent as to favor the development of ventral hernia or the formation of a fistulous tract. It is an accepted fact, that the object of drainage is to remove all those deleterious substances which, if allowed to remain within the peritoneal cavity or tion, and in many cases, if great care is not exercised,

it is a fruitful source for the entrance of septic matter.

Cases very frequently present themselves in which it is absolutely demanded for the safety of the patient. The cases in which it should be used are very numerous. I will mention the cases in which its use is necessary: 1. When pus or any other septic material has entered the peritoneal cavity before or during the operation. 2. When extensive adhesions have been severed, and there is an oozing surface. 3 . When hæmorrhage is feared, or when present and cannot be controlled. 4. Where there is malignant disease and danger of subsequent infection. 5. In cases where simple or tubercular peritonitis exists, or where there is a large accumulation of ascitic fluid. When it is deemed advisable to use drainage, the question then arises, what device should be employed for that purpose? It should be answered in the following way: If there is hæmorrhage that cannot be controlled by the application of ligatures or hæmostatic forceps, or by some of the many methods that we have at our command, the best plan is to pack the bleeding cavity with a strip of iodoform gauze and bring the end of it out at the lower angle of the wound. We then have tampon and drainage combined. At the expiration of forty-eight hours the gauze should be removed. If the bleeding has ceased and there is need for drainage, a small rubber or glass tube may be substituted for the gauze; if there is still a tendency to hæmorrhage, the gauze may be reintroduced and allowed to remain a few days longer. In cases where there is oozing that has been caused by the breaking of adhesions, or when pus has escaped into the peritoneal cavity, or when the large bowel has been ruptured, and the rent cannot be found, a glass tube open a both ends, and perforated on its sides with small round holes, should be used. The tube should be long enough to reach from the lower angle of the wound to the bottom of Douglas' pouch. The circumference of the tube should be determined by the quantity and consistency of the material that it is expected to convey out of the cavity; the smallest tube should be selected that will properiy carry the fluid. Care should be taken when the tube is introduced that it does not press upon a loop of intestine instead of upon the floor of the pelvis. Before inserting the tube a suture should be introduced and left unfastened, its object being to close the opening that remains after the removal of the drainage tube, otherwise a weak cicatrix might remain, that would favor the formation of ventral hernia. After the tube is in its proper position, and the abdominal wound closed and the usual antiseptic dressings ap- 
plied, a piece of rubber sheeting about one foot square, with a small hole in the centre of it, shonld be sprung over the tube in order to prevent the discharge soiling the dressings and infecting the wound. Aseptic absorbent cotton should then be placed over the opening in the tube to absorb the overflow, and over this the rubber sheeting is folded and retained in position with a safety pin. The most important point to be observed in the use of the drainage tube is frequent and thorough cleansing. No regular or fixed time can be set for emptying the tube, but a good rule should be to clean it whenever it is filled with fluid, no matter how short the interval. The fluid is best removed with a long nozzle uterine syringe, or a piece of rubber tubing attached to an ordinary syringe. When hamorrhage is indicated by the flow of pure blood from the tube, the blood must be constantly removed, as by keeping the bleeding surface dry coagulation is promoted, and the hamorrhage very often arrested. After the drainage tube has been in use for forty-eight hours, it becomes surrounded by the tissues, which are retained in that position by adhesive inflammation, therefore after that time, it is of very little if any use in draining the abdominal cavity. It will, however, drain the pelvis for a much longer time. The tube should be raised two or three inches out of the wound every day and turned completely around on its axis, and allowed to drop by its own weight, in order to prevent prolonged pressure on any one point, and also to prevent the organizing exudation from penetrating the lateral openings in the tube. The drainage tube should be allowed to remain until the discharge is roduced to 2 or 3 drachms of clear serum a day, this usually occurs within three days. After it has been removed, the free ends of the suture that was left for the closure of the drainage tube wound should be ticd.

\section{NON-MALIGNANT, NON-TUBERCULAR, NON- HYDATID CYSTIC DISEASE OF THE PERITONEUM.}

Read by invitation in the Section of Obstetries and Diseases of Women at the Forty-third Annual Meeting of the American Mredical Association. held at Detroit, Mich., June, 1892.

BY JAMES F. W. ROSS, M.D., OF TORONTO, ONT.

The subject selected is not likely to prove of interest to the general practitioner; it is not likely to open up any practical discussion, but it is of interest to the abdominal surgeon. The disease to be discussed is allied with acute peritonitis on the one hand and intermittent pelvic peritonitis on the other. The disease is cystic and yet inflammatory. It is therefore essential that we should consider the patholngy of cysts and the pathology of peritonitis. Authors vary in their classification of cysts, and yet agree in the main distinctive features of these interesting aberrations from a normal condition. For our present purpose we need but remember that we have epithelial cysts and endothelial cysts. Epithelial cysts are cysts of retention. Endothelial cysts are formed of the dilatation of cavities in connective tissue such as bursa, tendon sheaths and obstructed lymph chanmels (Zeigler). An inflamed bursa, from the inability of the serous lining to absorb the fluid poured into its interior, becomes a cyst, and if we would carry the analogy to what might be considered too great a length, the inflamed peritoneum, from the inability of its serous lining to absorb the fluid poured in to it, becomes a cyst. Newly formed or false membrane may surround fluid so that portions of the fluid become collected in sacs, and thus inflammatory cysts are formed. Pus is surrounded in this way in the abdomen, and pockets of encysted pus are shut off from the general peritoneum. The very same formation of pockets of encysted serum takes place subsequent to a peritonitis.

We have other cysts, such as the cysts of disintegration, and the cysts formed around foreign bodies, such as hydatids and blood cysts, but with these we have nothing to do at present. But I wish to mention the cysts that are found filled with lymph instead of serum, from the obstruction of the lymph channels; with blood instead of serum, from the intracystic rupture of one of the newly formed and delicate vessels; with air instead of serum, a condition that has been noticed by several good observers. The latter condition is a pathological curiosity. One case in which this condition was found has been well recorded in the London Pathological Society's Transactions, Vol. xxii. The patient suffered from a duodenal ulcer, and the supposition was that flatus escaped beneath the peritoneum and produced the condition. The cysts were like the cystic dilatations so frequently found hanging from the fimbriated extremity of a normal Fallopian tube, or from the tubules of Kobelt in a normal broad ligament. Some were as large as a hazel nut. They hung by a single pedicle. Some similar ones contained coagulated blood instead of air, but even in these, hollow spaces were found on section. The parietal as well as the visceral peritoneum was affected. In his discourse on the case I find the following, written by the author of the paper: "Were the cysts which contained the air of independent formation, or were they produced in any way by the emphysematous bubbles? Against the first view it must be urged that cysts of the peritoneum are hardly known; nothing of the kind is mentioned in the standard text-books." In the latter sentence you have my excuse for presenting to you such a subject. That I should thus early in my lifetime have met with two cases would seem to indicate that the disease is not of such rare occurrence. My friend, Dr. C. A. L. Reed, has promised to put two cases on record and to give his views regarding the etiology and treatment of the disease. We will return to the pathology after detailing the cases. My own cases were as follows:

Case 1.-Mrs. A. L. ret. 22, had evidently suffered from gonorrhcea two and a half years before she came under my observation. The history was that of intermittent pelvic peritonitis. When making a vaginal examination, I felt what I concluded was a urine-filled bladder, and asked her physician, Dr. Dawson, why she kept her bladder so full when she knew we required bladjer and rectum empty. She was at the time under the influence of an anæsthetic, so that I passed a catheter myself. No water came, and yet I felt sure that the fluid must be in the bladder. The wall surrounding the fluid was lax and not tense like the wall of an ovarian eyst. I then felt two pus tubes, one on each side of the uterus, and advised their removal. The collection of fluid was a mystery I was unable to solve.

On opening the abdomen three days after (namely, on November 4, 1891), I found peritoneal cysts looking like white grapes. Owing to the matting of bowel and omentum to some mass, I put the patient in Trendelenberg's position to facilitate the exploration. After pulling up the omentum a dark-colored cyst was found, occupying the position usually occupied by the bladder in front of the uterus. It had a grayish, semi-gangrenous appearance, and yet when 